\title{
ToyBox Study Malaysia: a feasibility study to improve healthy energy balance and obesity-related behaviour.
}

\author{
$\underline{\text { Sue Reeves }}^{1}$, B. K. Poh $^{2}$, Whye Lian Cheah ${ }^{3}$, Cecilia Essau ${ }^{1}$, Carolyn Summerbell ${ }^{4}$, \\ Denise $\mathrm{Koh}^{2}$, Julia Lee ${ }^{3}$, Ruzita Talib ${ }^{2}$ and Leigh Gibson ${ }^{1}$ \\ ${ }^{1}$ University of Roehampton, London, United Kingdom, \\ ${ }^{2}$ Universiti Kebangsaan, Kuala Lumpur, Malaysia, \\ ${ }^{3}$ Universiti Malaysia Sarawak, Kota Samarahan, Malaysia and \\ ${ }^{4}$ Durham University, Durham, United Kingdom
}

\section{Abstract}

Over the last two decades, levels of childhood overweight and obesity have increased considerably in Malaysia, such that the country now has the highest prevalence of obesity in Asia. The preschool years are a key time for establishing healthy behaviours; hence, there is a need for effective interventions aimed at early childhood. The ToyBox Study is an intervention to prevent obesity in preschoolers that has previously been conducted successfully in six different countries but to date not outside Europe. Therefore, we conducted a feasibility study to determine whether the ToyBox Study methodology could be successfully adapted and applied in Malaysia.

The ToyBox Study Malaysia was conducted in 15 kindergartens in Kuala Lumpur and suburbs in Selangor and 7 kindergartens in Sarawak (Borneo), Malaysia. All participating kindergartens were funded by Jabatan Kemajuan Masyarakat (KEMAS), the Community Development Department, Ministry of Rural and Regional Development.The existing ToyBox resources and questionnaires were translated into Bahasa Malaysia and the materials were adapted to suit local culture. Theory of change workshops and focus groups were conducted with parents, caregivers, teachers, cooks and government officers in order to help develop the implementation model. Train-the-trainer and teacher training sessions were organised before the study commenced.

The study was a randomised controlled trial that compared the ToyBox Study with kindergarten usual practice, over a period of one year. The ToyBox Study specifically targeted four energy balance related behaviours, namely; eating healthy snacks and meals, making water the preferred drink, increasing physical activity and reducing sedentary behaviour. Food frequency questionnaires were used to assess food intake. Accelerometry was used to measure physical activity. Weight, height and waist circumference were also measured. All measurements were made at baseline and post-intervention. Equipment such as soft mats and drinking water stations were supplied where needed. Parents, caregivers and teachers were provided with relevant materials, newsletters and tip cards and encouraged to participate and act as role models. They also completed project evaluation surveys.

If successful, it is envisaged that the ToyBox Study Malaysia will be adopted by other kindergartens in Malaysia, with the intention of helping Malaysian children and their families to achieve healthy energy balance related behaviours that will benefit their health and reduce obesity risk in the long-term.

\section{Acknowledgements}

The ToyBox Study Malaysia is funded by an MRC Newton-Ungku Omar Fund grant (MR/P013805/1). We thank KEMAS and the teachers, parents and children for their support with the project.

\section{Conflict of Interest}

There is no conflict of interest 